Ann. Biol. anim. Bioch. Biophys., I97I, 11 (3), 415-426.

\title{
CALCÉMIE, PHOSPHATÉMIE, MAGNÉSÉMIE ET GLYCÉMIE COMPARÉES DE LA MÈRE ET DU NOUVEAU-NTE CHEZ LES RUMINANTS DOMESTIQUES (VACHE, CHÈVRE, BREBIS)
}

\author{
J.-P. BARLET, M.-C. MICHEL, P. LARVOR et M. THÉRIEZ * \\ Station de Physiopathologie de la Nutrition, \\ * Station de Recherches sur l'Élevage des Ruminants, \\ Centre de Recherches de Clermont-Ferrand, I. N.R. A., \\ 63 - Saint-Genès-Champanelle
}

\section{RÉSUMÉ}

Chez 21 vaches, 35 brebis et ro chèvres nous avons mesuré la calcémie, la phosphatémie, la magnésémie et la glycémie dans les jours précédant et suivant la parturition. Ces 4 paramètres sanguins ont été également dosés, pendant les premiers jours de la vie chez les veaux, les chevreaux et les agneaux nés des animaux précédents. La stabilité de la calcémie et de la phosphatémie de ces jeunes animaux semble indiquer que, chez ces 3 espèces, les mécanismes de régulation du métabolisme phosphocalcique sont fonctionnels dès la naissance. Neuf veaux issus de vaches présentant le syndrome vitulaire ont une calcémie et une phosphatémie significativement inférieures à celles des veaux normaux, alors que leur magnésémie et leur glycémie ne sont pas modifiées. L'hypersécrétion de calcitonine accompagnant la parturition chez la Vache ne semble pas due à une élévation du taux de calcium ultrafiltrable.

Chez la Vache, la parturition est normalement associée à une hypocalcémie et à une hypophosphatémie, qui, lorsqu'elles deviennent très accentuées, aboutissent aux manifestations cliniques du syndrome vitulaire. Il était intéressant de voir si cette hypocalcémie et cette hypophosphatémie se manifestaient aussi chez la Chèvre et la Brebis parturientes. De plus, les seules études concernant la calcémie et la phosphatémie du Veau et de 1'Agneau avaient été réalisées in utero (SATO, I933; SYMoNDS et al., I966 ; BAWDEN et WoLKOFF, I967). Aucune mesure systématique de la calcémie, de la phosphatémie, de la magnésémie, et de la glycémie chez la mère et le nouveau-né au moment de la parturition n'avait été effectuée chez les Ruminants. Nous avons réalisé ce travail en essayant de mettre en évidence les particularités au niveau de ces paramètres chez les veaux issus de vaches présentant le syndrome vitulaire. 


\section{MATÉRIEL ET MÉTHODES}

Nous avons utilisé $2 \mathrm{I}$ vaches de races diverses (I5 Frisonnes, 2 Holstein, 2 Pie-Rouges et 2 Jersiaises) ayant eu une mise bas normale. Les prélèvements de sang étaient effectués chaque matin à heure fixe dans les jours précédant et suivant le vêlage et au moment même de celui-ci. Le sang des veaux était prélevé à la naissance et ensuite pendant 7 jours, chaque matin à heure fixe. Ces veaux recevaient uniquement le colostrum pendant cette période. Unn échantillon de sang a également été prélevé sur 23 vaches (2I Frisonnes, I Garonnaise et I Limousine) ayant présenté une crise d'hypocalcémie vitulaire dans les $24 \mathrm{~h}$ après la mise bas, et en même temps, sur 9 de leurs veaux.

Chez Io chèvres de race Alpine le sang a été prélevé 6 jours avant la mise bas, et chez les chèvres et leurs chevreaux, au moment de celle-ci et pendant les 6 jours suivants à heure fixe.

Chez 35 brebis de race Limousine, nous avons prélevé de la même façon du sang 7 jours avant. l'agnelage, et chez ces brebis et leurs agneaux, au moment même de l'agnelage et 7 jours plus tard.

Sur tous ces animaux le sang était recueilli sur héparine par ponction d'une veine jugulaire, immédiatement centrifugé à $5000 \mathrm{~g}$, pendant $10 \mathrm{mn}$, et le plasma recueilli.

Le calcium total et le magnésium plasmatiques étaient dosés par spectrophotométrie d'absorption atomique (Appareil Perkin-Elmer 303). Le plasma était ultrafiltré par passage à travers une membrane "U. M. 20 E Diaflo " qui arrête les molécules d'un poids supérieur à 20 ooo (BLATr et al., r967), et le calcium du filtrat était mesuré de la même façon que le calcium total.

Le phosphore minéral et le glucose étaient dosés, après défécation des protéines du plasma. à l'acide trichloracétique, à l'aide d'un auto-analyseur Technicon. La mesure du phosphore minéral est effectuée par une méthode colorimétrique à l'acide molybdique (KALCKAR, I947), et celle du glucose par la glucose-oxydase (MIcher, I97 I).

\section{RÉSULTATS}

Chez la Chèvre, comme chez la Vache, la parturition est associée à une hypocalcémie, à une hypophosphatémie, à une hypermagnésémie et à une hyperglycémie

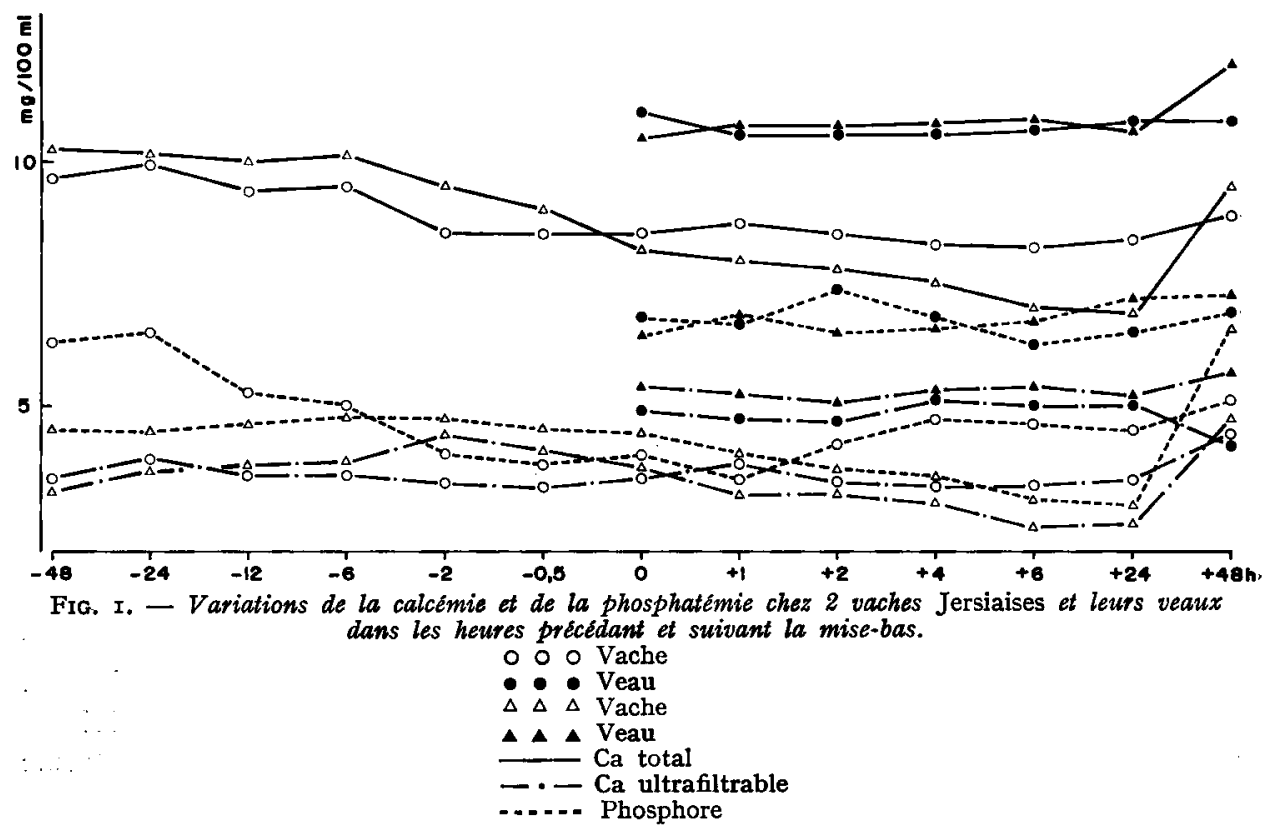




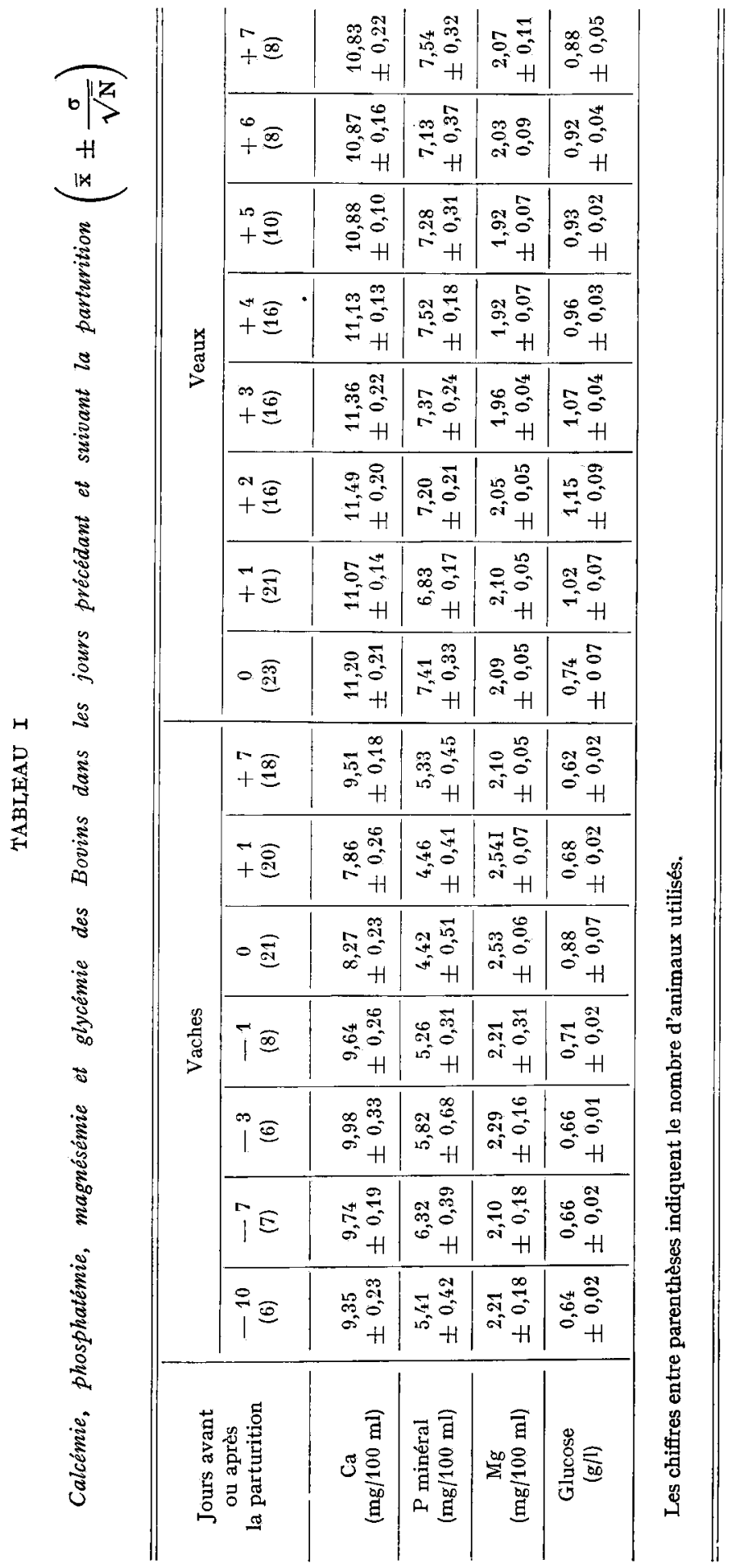




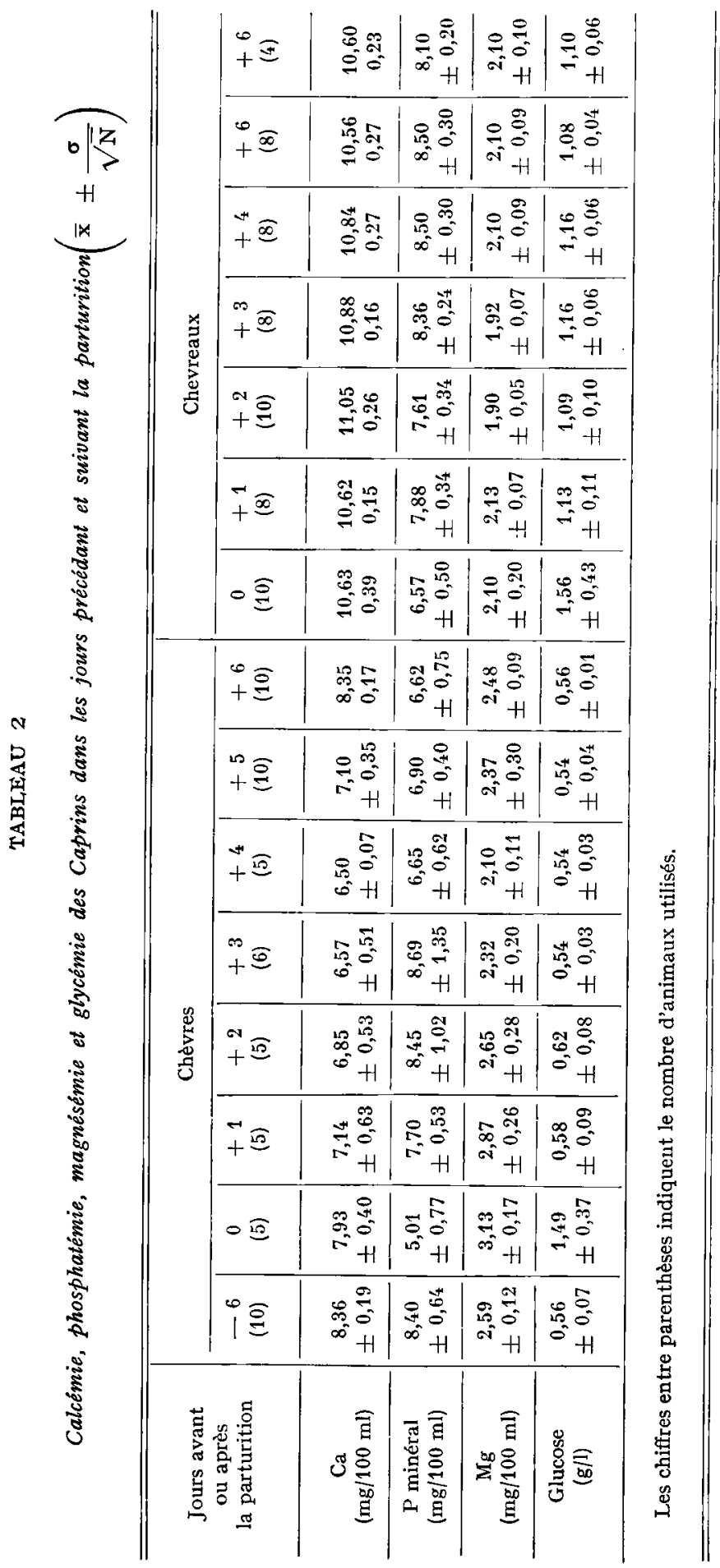


significatives. Aucune de ces variations ne se retrouve de façon significative chez la Brebis (tabl. I, 2 et 3 ).

TABLEAU 3

Calcémie, phosphatémie, magnésémie et glycémie de la Brebis et de l'Agneau dans les jours précédant et suivant la parturition $\left(\overline{\mathbf{x}} \pm \frac{\sigma}{\sqrt{ } \mathbf{N}}\right)$

\begin{tabular}{c|c|c|c|c|c}
\hline \hline \multirow{2}{*}{$\begin{array}{c}\text { Jours avant ou } \\
\text { après l'agnelage }\end{array}$} & \multicolumn{3}{|c|}{ Brebis } & \multicolumn{2}{|c}{ Agneaux } \\
\cline { 2 - 6 } & $-7(35)$ & $0(35)$ & $+7(22)$ & $0(55)$ & $+7(34)$ \\
\hline $\begin{array}{c}\mathrm{Ca} \\
(\mathrm{mg} / 100 \mathrm{ml})\end{array}$ & $9,71 \pm 0,12$ & $9,43 \pm 0,15$ & $9,57 \pm 0,20$ & $11,25 \pm 0,11$ & $11,99 \pm 0,12$ \\
\hline $\begin{array}{c}\mathrm{P} \text { minéral } \\
(\mathrm{mg} / 100 \mathrm{ml})\end{array}$ & $3,40 \pm 0,16$ & $3,82 \pm 0,15$ & $4,94 \pm 0,42$ & $6,94 \pm 0,14$ & $9,47 \pm 0,13$ \\
\hline $\begin{array}{c}\mathrm{Mg} \\
(\mathrm{mg} / 100 \mathrm{ml})\end{array}$ & $1,98 \pm 0,03$ & $1,92 \pm 0,01$ & $1,91 \pm 0,04$ & $1,91 \pm 0,03$ & $1,78 \pm 0,02$ \\
\hline $\begin{array}{c}\mathrm{Glucose} \\
(\mathrm{g} / \mathrm{l})\end{array}$ & $0,56 \pm 0,01$ & $0,58 \pm 0,02$ & $0,59 \pm 0,02$ & $1,04 \pm 0,04$ & $1,06 \pm 0,01$ \\
\hline
\end{tabular}

Les chiffres entre parenthèses indiquent le nombre d'animaux utilisés.

Chez les Ovins le calcium ultrafiltrable représente une fraction du calcium total significativement plus importante que chez les Bovins. Nous n'avons cependant pas pu mettre en évidence de variations significatives du taux de calcium ultrafiltrable au moment de la parturition chez la Brebis ou chez la Vache (tabl. 4, fig. I).

Chez la Vache, au moment de la parturition, il existe une corrélation négative entre la calcémie et la magnésémie (pour 20 D.L., $r=-0,5 \mathrm{I} 6$, pour $p \leqslant 0,05$, $r=-0,444$ ). Cette corrélation significative ne se retrouve pas $24 \mathrm{~h}$ plus tard (pour I8 D.L., $r=-0,439)$.

La calcémie et la phosphatémie du Veau, du Chevreau et de l'agneau sont supérieures à celles de leurs mères de façon significative, aussi bien à la naissance que 6 ou $^{\circ} 7$ jours plus tard (tabl. I, 2 et 3 ). Comme le calcium total, le calcium ultrafiltrable du Veau de $o$, de $I$ et de 7 jours et de l'Agneau de 0 et de 7 jours est significativement supérieur à celui de leurs mères (tabl. 4).

Alors que la magnésémie du Veau et du Chevreau est significativement supérieure à celle de leurs mères à la naissance, au même moment, cette différence ne se retrouve pas entre l'agneau et la Brebis (tab1. $x, 2$ et 3 ).

La glycémie de la Vache parturiente est significativement supérieure à celle du Veau nouveau-né ; 24 h plus tard c'est la glycémie du Veau qui est significativement plus élevée que celle de la Vache normale. Il n'existe pas de différence significative entre la glycémie de la Chèvre parturiente et de son Chevreau, alors, que dès la naissance, la glycémie de 1'Agneau est significativement supérieure à celle de la Brebis (tabl. I, 2 et 3 ). 


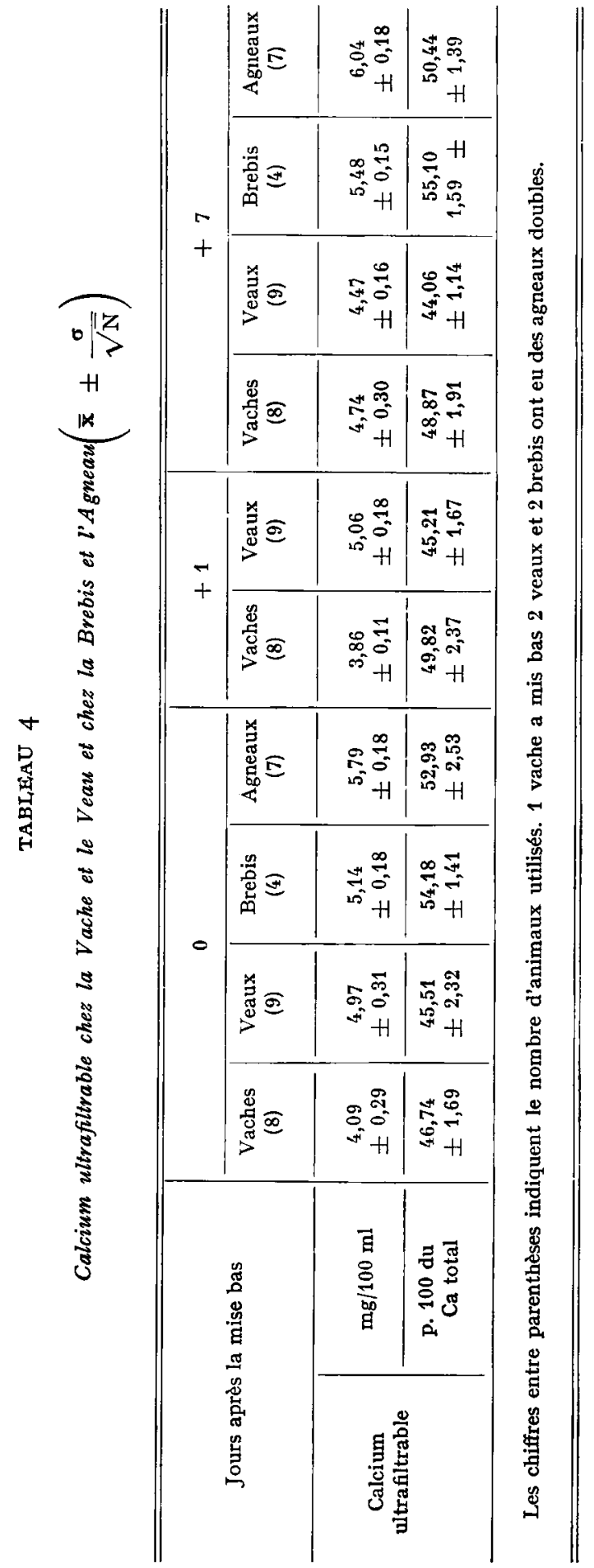


Chez les vaches atteintes d'hypocalcémie vitulaire, $24 \mathrm{~h}$ après la parturition, l'hypocalcémie et l'hypophosphatémie sont plus prononcées, de façon hautement significative, que chez les vaches normales. Il n'existe pas de différence significative entre la légère hypermagnésémie des animaux parturients normaux et hypocalcémiques ; par contre, l'hyperglycémie est significativement plus prononcée chez ceux-ci $24 \mathrm{~h}$ après le vêlage que chez les animaux normaux. Les veaux issus de vaches atteintes d'hypocalcémie vitulaire ont une calcémie et une phosphatémie significativement inférieures à celles des veaux issus de vaches normales (tabl. I et 5).

\section{TABLEAU 5}

Calcémie, phosphatémie, magnésémie et glycémie au cours du syndrome vitulaire

$$
\left(\overline{\mathbf{x}} \pm \frac{\sigma}{\sqrt{\mathrm{N}}}\right)
$$

\begin{tabular}{|c|c|c|c|}
\hline & Vaches (23) & Veaux (9) & Chèvres (2) \\
\hline $\begin{array}{c}\mathrm{Ca} \\
(\mathrm{mg} / 100 \mathrm{ml})\end{array}$ & $4,62 \pm 0,27$ & $9,83 \pm 0,48$ & $\begin{array}{l}3,10 \\
2,97\end{array}$ \\
\hline $\begin{array}{c}\text { P minéral } \\
(\mathrm{mg} / 100 \mathrm{ml})\end{array}$ & $1,98 \pm 0,32$ & $6,05 \pm 0,40$ & $\begin{array}{l}2,43 \\
4,12\end{array}$ \\
\hline $\begin{array}{c}\mathrm{Mg} \\
(\mathrm{mg} / 100 \mathrm{ml})\end{array}$ & $2,68 \pm 0,25$ & $2,11 \pm 0,19$ & $\begin{array}{l}2,17 \\
2,12\end{array}$ \\
\hline $\begin{array}{l}\text { Glucose } \\
(\mathrm{g} / \mathrm{l})\end{array}$ & $0,879 \pm 0,05$ & $0,983 \pm 0,05$ & $\begin{array}{l}1,370 \\
1,833\end{array}$ \\
\hline
\end{tabular}

Les chiffres entre parenthèses indiquent le nombre d'animaux utilisés

Chez 2 chèvres, nous avons observé un syndrome caractérisé par une paralysie partielle au niveau des membres postérieurs, une hypothermie (température corporelle inférieure à $37^{\circ} \mathrm{C}$ ), une hypocalcémie associée à une hypophosphatémie et à une hyperglycémie (tabl. 5). Comme dans le syndrome vitulaire de la Vache, une injection intraveineuse de glutamate de calcium amenait une guérison rapide.

\section{DISCUSSION}

Contrairement à ce qui se passe chez la Vache et la Chèvre, il n'apparaît chez la Brebis, à la mise bas, ni hypocalcémie, ni hypophosphatémie significatives. Parmi les 35 brebis que nous avons utilisées, 20 ont donné naissance à 2 agneaux et I 5 à I agneau. Bien qu'au moment de l'agnelage la calcémie des mères d'agneaux doubles soit légèrement inférieure à celle des mères d'agneaux simples $(9,26 \pm 0,20$ contre $9,70 \pm 0,20 \mathrm{mg} /$ roo $\mathrm{ml}$ ) cette différence n'est pas significative. La phosphatémie 
des mères d'agneaux doubles est d'ailleurs légèrement supérieure, de façon non significative, à celle des mères d'agneaux simples $(3,96 \pm 0,30$ contre $3,63 \pm 0,21 \mathrm{mg} /$ Ioo $\mathrm{ml}$ ). Il n'existe pas non plus de différence significative entre ces deux groupes de brebis au niveau de la magnésémie et de la glycémie.

En ce qui concerne ces 4 paramètres sanguins chez les agneaux, il n'existe, à o ou à 7 jours, aucune différence significative entre les agneaux nés simples ou jumeaux, ou entre les mâles et les femelles. Le même résultat se retrouve chez les Bovins : 2 vaches ayant mis bas chacune 2 jumeaux ont eu un comportement tout à fait identique à celui des vaches analogues ayant eu un seul veau. Les paramètres sanguins de ces 4 jumeaux ne sont pas significativement différents de ceux des veaux uniques.

On sait depuis longtemps que, chez les Bovins, la calcémie foetale est supérieure à la calcémie maternelle (SATo, I933). De même, chez les Ovins, la calcémie et la phosphatémie du fotus sont supérieures à celles de la mère : cette hypercalcémie et cette hyperphosphatémie fœales ont été respectivement attribuées à un transfert actif du calcium à travers le placenta (BAWDEN, WOLKOFF et FLOWERS, r965) et à une insuffisance parathyroïdienne du fotus à terme (Sмrтr et al., 1969). Si les travaux effectués à 1'aide du ${ }^{45} \mathrm{Ca}$ tendent à prouver le transfert actif du calcium à travers le placenta, notamment chez la Femme, la Vache et la Ratte (Comar, I956), un éventuel hypoparathyroïdisme chez le fotus à terme parait beaucoup plus discutable. Bien que notre travail ne permette pas d'éliminer cette éventualité, elle paraît peu probable, puisque la calcémie de l'Agneau à la naissance est significativement supérieure à celle de la Brebis et que cette différence significative persiste pendant la première semaine de vie. D'ailleurs, chez le Rat, la calcémie fœetale pouvant varier indépendamment de celle de la mère, le fotus doit avoir ses propres mécanismes régulateurs de la calcémie (Jost et al., I963). De plus, les parathyroïdes prélevées sur le fotus ovin de 30 jours paraissent tout à fait fonctionnelles (SCOTHORNE, I964).

Chez le Veau nouveau-né, le taux de calcium ultrafiltrable, comme la calcémie est supérieur à celui observé au même moment chez la Vache; nous avons retrouvé une différence analogue entre 1'Agneau nouveau-né et la Brebis. Une observation similaire avait déjà été faite chez cette espèce après le premier mois de gestation et, chez la Femme, au moment de 1'accouchement (Delivoria-Papadopoulos et al., I967). Chez la Vache parturiente, les variations du taux de calcium ultrafiltrable sont parallèles à celles de la calcémie. Ce résultat diffère de celui obtenu par d'autres auteurs sur une vache Jersiaise chez laquelle il avait été observé, $6 \mathrm{~h}$ avant le vêlage, une augmentation de l'ordre de $80 \mathrm{p}$. Ioo du taux de calcium ultrafiltrable, suivie d'une chute du même ordre I2 h plus tard (BACH et MESSERvy, I969). Ceci semble indiquer que ce n'est pas une élévation du taux de calcium ultrafiltrable qui est responsable de l'hypersécrétion de calcitonine survenant à ce stade physiologique (BARLET, I969 $a$; CARE et al., I970).

Chez la Vache parturiente, il existe une corrélation négative entre la calcémie et la magnésémie. Cette corrélation ne se retrouve pas chez la Brebis ou chez la Chèvre. Dans l'espèce humaine, il se produit une légère hypocalcémie au moment de l'accouchement (PAUPE et al., I96r), mais il existe une corrélation positive entre la baisse de la calcémie et la baisse de la magnésémie observée chez chaque individu (RICE, SCHNEIDER et WEED, 1969). Chez la Vache, la parturition est accompagnée d'une élévation significative du taux de parathormone, proportionnelle à l'hypo- 
calcémie observée (MAYER et al., I969). Il est possible que cet hyperparathyroïdisme transitoire soit responsable de cette légère hypermagnésémie (MAC INTYRE, Boss et Throughton, I963).

Les veaux issus de vaches présentant le syndrome vitulaire $24 \mathrm{~h}$ après la mise bas ont, à ce moment, une calcémie et une phosphatémie significativement inférieures à celle des veaux issus de vaches normales. Plusieurs explications sont possibles :

On peut supposer que chez les vaches à fièvre vitulaire, l'hypersécrétion de calcitonine qui est à l'origine du syndrome (BARLET, I969a) entraîne un passage accru de cette hormone à travers le placenta, avec hypocalcémie fœtale. Cependant, la durée de vie de 1'hormone étant très brève (40 mn chez le Porc) (WEST, O'RIORdAN et CARE, I969), la calcémie des veaux devrait redevenir normale rapidement après la naissance, ce qui n'est pas le cas. De plus, le franchissement de la barrière placentaire par la calcitonine, bien que non étudiée chez le Ruminant, est inexistant chez le Rat (Garei, Milhaud et Sizonenko, I969). Enfin, la calcitonine endogène (CARE, Webster et Duncan, I966) ou exogène (BARLET, I968) est inactive chez le jeune veau.

L'hypocalcémie maternelle pourrait diminuer le transfert de calcium à travers le placenta. En effet, 1'administration de calcitonine à la Ratte gestante fait baisser la calcémie des fotus (GAREI, MirHaUd et Jost, I968; WezEMAN, I969) sans qu'il $\mathrm{y}$ ait passage hormonal à travers le placenta (GAREL, MriHaud et Sizonenko, I969). Cependant, cette explication n'est pas satisfaisante puisque l'hypocalcémie n'apparaît chez la Vache que quelques heures après la parturition (BARLET, r969 $b$ ).

Enfin, selon une théorie récente (MAC INTYRE, I969), la calcitonine, non seulement inhiberait le catabolisme osseux (MILHAUd, PÉRAuri et MoukHTAR, I965), mais interviendrait au niveau de la pompe à calcium de la cellule. Il est possible que les transferts calciques à travers le placenta soient ralentis de cette façon.

Il ne faut cependant pas exclure la possibilité que les veaux de ces vaches atteintes d'hypocalcémie vitulaire aient reçu moins de colostrum que les témoins, soit par suite d'une baisse de production de la mère, soit par suite de difficultés à la tétée chez une vache gardant difficilement une position normale. Dans ce cas la légère hypocalcémie du veau résulterait du jeûne (HERD, I966).

Chez la Vache et la Chèvre, où l'on observe une hypocalcémie à la parturition, celle-ci est associée à une hyperglycémie. Cette hyperglycémie ne se manifeste pas chez la Brebis, chez laquelle il n'y a pas non plus d'hypocalcémie. L'hyperglycémie associée à la parturition ou au syndrome vitulaire ne résulte pas d'une hyperglucagonémie (BARLET, I970). Il est possible qu'elle soit due à une hypoinsulinémie liée à l'hypocalcémie (LITTLEDIKE, WITZEL et WHIPP, I968).

Chez aucune de ces 3 espèces utilisées, nous n'avons pu mettre en évidence une corrélation entre la glycémie de la mère et du nouveau-né. La glycémie du nouveau-né humain paraît cependant réglée par celle de la mère (CoL'TAR' et al., I969) et, chez la Brebis, il existe une corrélation positive entre la glycémie maternelle et fotale du $5^{8}$ au $\mathrm{I}^{88^{\mathrm{e}}}$ jour de gestation (SHELLEY, I96o). Il est possible que l'anoxie transitoire survenant à la naissance perturbe considérablement la glycémie du nouveau-né (MANN, I970).

A la naissance, la glycémie du Veau est significativement inférieure à celle observée 24 h plus tard : il a été montré que, chez le Rat, à partir de quelques heures avant 
la naissance, le fotus est capable de mobiliser une partie du glycogène hépatique, mobilisation qui se poursuit d'ailleurs après la naissance (DAwKINs, I963). Un phénomène analogue pourrait se produire pendant les premières heures de la vie du Veau; l'Agneau semble d'ailleurs utiliser ses réserves hépatiques en glycogène très rapidement à la naissance (SHELLEY, I960).

Chez 2 chèvres, produisant quotidiennement plus de 4,5 1 de 1ait, nous avons observé, respectivement 4 et 33 jours après la mise bas, un syndrome cliniquement et biochimiquement analogue au syndrome vitulaire de la vache. Ceci indique que, chez la Chèvre, peuvent survenir des troubles du métabolisme phosphocalcique probablement liés à une hypersécrétion de calcitonine, puisque caractérisés par une hypocalcémie et une hypophosphatémie.

\title{
CONCLUSION
}

La Vache et la Chèvre, dont la parturition est associée à une hypocalcémie et à une hypophosphatémie significatives se comportent d'une façon différente de la Brebis chez laquelle, à ce stade physiologique, nous n'avons pu mettre en évidence ni hypocalcémie ni hypophosphatémie. L'hypersécrétion de calcitonine qui accompagne le vêlage chez la Vache ne semble pas due à une augmentation du taux de calcium ultrafiltrable. Le Veau, le Chevreau et l'Agneau ont une calcémie et une phosphatémie qui dès la naissance, sont supérieures à celles de leurs mères et qui restent stables pendant la première semaine de leur vie. Les veaux nés de vaches présentant le syndrome vitulaire ont, pour une cause inconnue, une calcémie et une phosphatémie significativement inférieures à celles des veaux issus de vaches normales.

Reçu pour publication en mars 1971.

\section{REMERCIEMEN'TS}

Nous remercions M. Journet (Station de Recherches sur l'Élevage) et M. Champredon (Station d'Étuđe des Métabolismes) qui nous ont fourni la plupart des Bovins et Caprins utilisés pour ce travail. M. le docteur Roche (64 - Lembeye) a aimablement accepté de recueillir le sang des vaches présentant le syndrome vitulaire et de leurs veaux.

\section{SUMMARY}

\author{
BLOOD CALCIUM, PHOSPHORUS, MAGNESIUM AND SUGAR \\ IN MOTHERS AND THEIR NEWBORN IN DOMESTIC RUMINANTS \\ (COW, GOAT AND EWE)
}

There are significant reductions in blood calcium and phosphorus at parturition in cows and goats. In those species these falls may be great and may give rise to clinical signs of the parturient paresis syndrome. In ewes we have been unable to demonstrate a fall in either calcium or phosphorus in blood at lambing. In cows there is no significant variation in the content of 
ultrafiltrable calcium in the hours before or after calving. This excludes the possibility of stimulation of secretion of calcitonin by an increase in ultrafiltrable calcium in cows at this physiological stage. Blood calcium and phosphorus in calves, kids and lambs are higher from birth than values in their dams and are remarkably stable for the first week of life. Calves from cows which develop clinically low blood calcium at calving have less blood calcium and phosphorus than calves from healthy cows. The cause of this small reduction in blood calcium and phosphorus could be either an effect of calcitonin on transfer of calcium across the placenta or deficient feeding of the animals during the first 24 hours of life.

\section{RÉFÉRENCES BIBLIOGRAPHIQUES}

BACH S. J., Messervy A., r969. Observations on the diffusible calcium fraction in the serum of cows during oestrus and during parturition. Vet. Record, 84, $210-213$.

BARLet J.-P., 1968. Effets de la thyrocalcitonine sur la calcémie et la phosphatémie du veau, C. $R$. Acad. SC., D, 266, 395-398.

BARLET J.-P., I 969 a. Mise en évidence d'un facteur hypocalcémiant et hypophosphatémiant dans le plasma des vaches laitières; variations du taux de ce facteur au moment du vêlage et dans les cas de syndrome vitulaire. C. R. Acad. Sc., D, 210, 1864-I867.

BARIET J.-P., r $969 b$. Variations de la calcémie et de la phosphatémie chez la vache laitière au moment du vêlage : rôle probable de la calcitonine dans l'étiologie du syndrome vitulaíre. Rech. Vétér., 2, 93-Ioo.

BARLET J.-P., r97o. Effet du glucagon ou de l'arginine sur la calcémie et la phosphatémie de la vache laitière. C. R. Acad. Sc., D, 210, I161-I164.

Bawden J. W., Wolkoff A. S., Flowers C. E., 1956. Maternal fetal blood calcium relationships in sheep. Obstet. Gynec., 25, 548-552.

Blatt W. F., Hudson B. G., Robinson S. M., Zipilivan E. M., 1967. Fractionation of protein solutions by membrane partition chromatography. Nature, 216, 5 II-513.

Care A. D., Webster D., Duncan T., 1966. Species variation to thyrocalcitonin. Excerpta Medica International Congress Series, 120, I3.

Care A. D., Bates R. F. L., Philitippo M., Lequin R. M., Hackeng W. H. L., Barlet J.-P., Larvor P., I970. Stimulation of calcitonin release from bovine thyroid by calcium and glucagon. J. Endocr., 48, 667-668.

Coltart T. M., Beard R. W., Turner R. C., Oakley N. W., I969. Blood glucose and insulin relationships in the human mother and foetus before onset of labour. British Med. J., 4, I7-19.

Comar C. L., 1956. Radiocalcium studies in pregnancy. Ann. N. Y. Acad. Sci., 64, 281-298.

Dawkins M. J. R., rg63. Glycogen synthesis and breakdown in fetal and newborn rat liver. Ann. N. Y. Acad. Sci., 31, 203-21 I.

Delivoria-Papadopoulos M., Battaglia C. F., Bruns P. D., Meschia G., ig67. Total, proteinbound and ultrafiltrable calcium in maternal and fetal plasmas. Amer. J. Physiol., 213, 363-366.

Garel J.-M., Milhaud G., Jost A., r968. Action hypocalcémiante et hypophosphatémiante de la thyrocalcitonine chez le fotus de rat. C. $R$. Acad. Sc., D, 267, 344-347.

Garel J.-M., Milhaud G., Sizonenko P., 1969. Thyrocalcitonine et barrière placentaire chez le rat. C. R.Acad. Sc., D, 269, 1785-1787.

HERD R. J. P., I966. Fasting in relation to hypocalcaemia and hypomagnesaemia in lactating cows and ewes. Australian Vet. J., 42, 269-272.

Jost A., Pic P., Maniey J., Legrand C., 1963. Action du propyl-thiouracile donné à la ratte gestante sur les parathyroïdes et sur la calcémie des fotus. Remarques sur la physiologie des parathyroides. Acta Endocrinol., 43, 618-624.

Kalckar H. M., 1947. The enzymatic synthesis of purine ribosides. J. Biol. Chem., 167, 477-486.

IrTtledike E. T., Witzel D. A., WhipP S. C., I968. Insulin : evidence for inhibition of release in spontaneous hypocalcemia. Proc. Soc. Exp. Biol. Med., 129, 135-139.

Mac INTYRe I., 1969. In Calcitonin 1969. Proceedings of the second international Symposium, 1-13, W. Heinemann, Londres.

Mac Intyre I., Boss S., Troughton V. A., I963. Parathyroid hormone and magnesium homeostasis. Nature, 108, 1058-106o.

MANN L. I., I97o. Effects in sheep of hypoxia on levels of lactate, pyruvate, and glucose in blood of mothers and fetus. Pediat. Res., 4, 46-54.

Mayer G. P., Ramberg C. F., Kronfeld D. S., Buckle R. M., Sherwood L. M., Aurbach G. D., PotTs J. T., I969. Plasma parathyroid hormone concentration in hypocalcemic parturient cows. Amer. J. Vet. Res., 80, 1587-1597. 
Michel M. C., 197r. Dosage du glucose et du phosphore minéral dans le sang à l'autoana lyseur Tech nicon. Ann. Biol. Anim. Bioch. Biophys. (sous presse).

Milhaud G., Pérault A. M., Moukhtar M. S., 1965. Étude du mécanisme de l'action hypocalcémiante de la thyrocalcitonine. C. R. Acad. Sc., D, 261, 813-8 6 .

Paupe J., Colin J., Politis E., Lelong M., I96r. Variations physiologiques de la calcémie chez la mère au moment de l'accouchement, dans le cordon, et chez le nouveau-né. Biol. Néonat., 3, 357-378.

Rice B. F., Schneider G., WeEd J., 1969. Serum calcium and magnesium concentration during early labor and the post partum period. Am. J. Obst. Gynec., 104, I I59-1 162.

SATo Y., I933. Studies on the metabolism of calcium in the fotus. Trans. Soc. Pathol. Japan., 28, 257-262.

Scothorne R. J., I964. Functional capacity of fetal parathyroid glands with reference to their clinical uses as homografts. Ann. N. Y. Acad. Sci., 120, 669-672.

Shelley H. J., I960. Blood sugars and tissue carbohydrate in foetal and infant lambs and rhesus monkeys. J. Physiol., 153, 527-552.

Symonds H. W., Manston R., Payne J.-M., Samson B. F., Ig66. Changes in the calcium and phosphorus requirements of the dairy cow at parturition with particular reference to the amounts supplied to the foetus in utero. Br. Vet. J., 122, 196-200.

West T. E. T., O'Rlordan J. L. H., CARE A. D., I969. The clearance of homologous calcitonin. J. Endocr., 45, 495-504.

WezEMAN F. H., I969. Effect of maternally administered thyrocalcitonin on fetal calcium levels. Fed. Proc., 28, 384 . 